\title{
Problems of Clustering of Radiogalaxies
}

\author{
Włodzimierz Godłowski ${ }^{1}$, Agnieszka Pollo ${ }^{2,3}$ and Jacek Golbiak ${ }^{4}$ \\ ${ }^{1}$ Institute of Physics, Opole University, Oleska 48, 45-052 Opole, Poland \\ email: godlowski@uni.opole.pl \\ ${ }^{2}$ Astronomical Observatory, Jagellonian University, Orla 171, 30-244 Krakow, Poland \\ ${ }^{3}$ National Centre for Nuclear Research, Hoza 69, 00-689 Warszawa \\ email: apollo@camk.edu.pl \\ ${ }^{4}$ Institute of Philosophy of Nature and Natural Sciences, John Paul II Catholic University of \\ Lublin, Al. Racawickie 14, 20-950 Lublin, Poland \\ email: jgolbiak@kul.lublin.pl
}

\begin{abstract}
We present the preliminary analysis of clustering of a sample of 1157 radio-identified galaxies from Machalski \& Condon (1999). We found that for separations $2-15 h^{-1} \mathrm{Mpc}$ their redshift space autocorrelation function $\xi(s)$ can be approximated by the power law with the correlation length $\sim 3.75 h^{-1} \mathrm{Mpc}$ and slope $\gamma \sim 1.8$. The correlation length for radiogalaxies is found to be lower and the slope steeper than the corresponding parameters of the control sample of optically observed galaxies. Analysis the projected correlation function $\Xi(r)$ displays possible differences in the clustering properties between active galactic nuclei (AGN) and starburst (SB) galaxies.
\end{abstract}

Keywords. Radiogalaxies, autocorrelation function

Clustering of the radiogalaxies was first detected by Peacock \& Nicholson (1991) in the redshift survey of 329 galaxies with $z<0.1$ and radio fluxes $S(1.4 \mathrm{GHz})>500 \mathrm{mJy}$ (hereafter PN91). They found that the redshift space correlation function of these radiogalaxies could be fitted by $\zeta=\left[s / 11 h^{-1} \mathrm{Mpc}\right]^{-1.8}$ where $s$ is the galaxy-galaxy separation in the redshift space. Later Peacock (1997) analyzed the sample of 451 radio identified galaxies selected from the LCRS (Shectman et al. (1996)) and NVSS (Condon et al. (1998)) surveys. Using the projected correlation function $\Xi(r)=\int \xi\left[\left(r^{2}+x^{2}\right)^{0.5}\right] d x$ he found that for the optical galaxies the correlation length was $\sim 5 h^{-1} M p c$ while the correlation length for the radio-loud subsample was $\sim 6.5 h^{-1} M p c$. Peacock (1997) suggested that these differences resulted from the fact that his sample was dominated by starburst (SB) galaxies while the majority of the PN91 sample were luminous active galactic nuclei (AGNs). In the present paper we analyze clustering of radiogalaxies using the sample of 1157 galaxies selected from the LCRS and NVSS by Machalski \& Condon (1999).

We measured angular and spatial autocorrelation functions $w(\theta)$ and $\xi(s)$ both for optical galaxies and radiogalaxies The measurement for optical galaxies remains very similar to that obtained by Tucker et al. (1997). The angular correlation function of radiogalaxies is characterized by the slope $\epsilon=\gamma-1=0.97 \pm 0.10$ for ALL the sample, with $\epsilon=1.13 \pm 0.14$ for AGNs and $\epsilon=0.86 \pm 0.14$ for SB galaxies. The $w(\theta)$ deviates from the power law on a scale $0.37^{\circ}$, which is lower than the value obtained for optical LCRS galaxies $\left(0.54^{\circ}\right)$. The redshift space correlation function for radiogalaxies can also be approximated by a power law; the measured correlation lengths are $3.75 h^{-1} \mathrm{Mpc} \pm 0.4$ for all classes of radio sources (ALL, AGN and SB). However, slopes are different in all cases: we obtain the value of $\gamma=1.76 \pm 0.11$ for a general sample of radiogalaxies, $2.39 \pm 0.34$ for AGNs and $1.66 \pm 0.15$ for SB galaxies The redshift space correlation function displays the same feature as the angular correlation function: a different slope for optical and radiogalaxies, and inside a radio-loud sample - a different slope for AGNs 


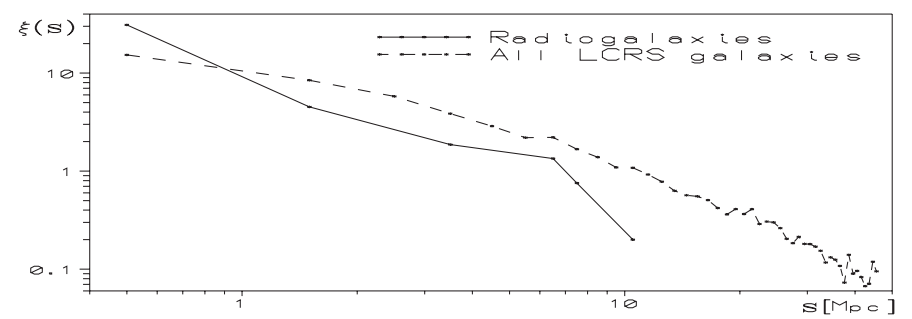

Figure 1. Redshift space autocorrelation function $\xi(r)$ for optical galaxies and radiogalaxies (for historical reasons $h=0.5$ is assumed).
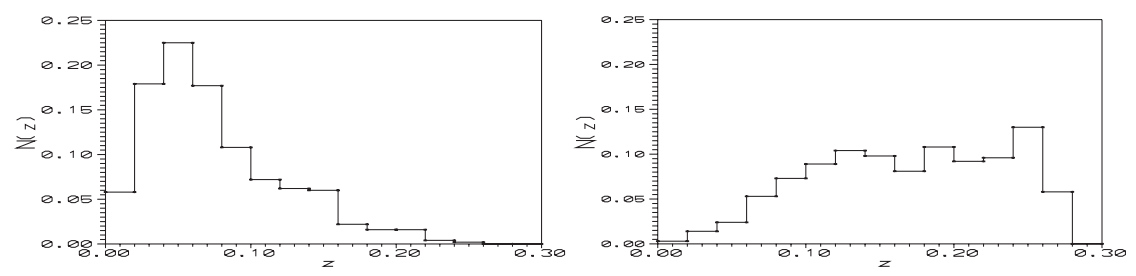

Figure 2. Redshift distribution of radiogalaxies: SB (left panel) and AGN (right panel).

and SB galaxies. In general, the value of $\gamma$ for radiogalaxies is higher than that found for optical galaxies: $\gamma=1.52 \pm 0.04$ (see Fig. 1). The analysis of the projected autocorrelation function $\Xi(r)$ suggests that the correlaton length is higher for AGNs than for SB galaxies. In the same time, the measured correlation length for AGNs is lower than that obtained for optical LCRS galaxies. In our opinion, the differences between all the samples are a mixed effect of two factors: a selection bias (mainly different redshift distributions for AGNs and SB galaxies, see Fig. 2) and different environments of AGN and SB galaxies.

Our main result is that for separations between $2-15 h^{-1} \mathrm{Mpc}$ autocorrelation function $\xi(s)$ for radiogalaxies can be approximated by the power law with slope $\gamma \sim 1.8$ and correlation length $\sim 3.75 h^{-1} \mathrm{Mpc}$. The measurements of the projected autocorrelation function $\Xi$ suggest that the correlation length for AGNs is higher than that for SB galaxies. All approaches to the measurement of the correlation function show differences in the slope coefficients between AGN and SB radiogalaxies. This result can be interpreted as following: AGN are radio sources of type FRI and FRII. We have no information whether a particular AGN belongs to the type FRI or FRII. However, it is clear that a significant number of the radio sources classified as AGNs is connected to elliptical galaxies located in the centers of galaxy clusters. In contrast, SB are mostly spiral galaxies, and they are located more often in the outer parts of clusters or even on the borders of filaments where processes of galaxy formation related to the starburst processes are stronger in the local Universe.

\section{References}

Condon, J. J., et al. 1998, AJ, 115, 1693

Machalski, J. \& Condon, J. J. 1999, ApJS, 123, 21

Peacock, J. A. \& Nicholson, D. 1991, MNRAS, 253, 307

Peacock, J. A. in "The Most Distant Radio Galaxies" \& Amsterdam, 1997, Royal Netherlands Academy of Arts and Sciences, eds H. J. A. Rttgering, et al. 1999, p. 377.

Shectman, S. A, et al. 1996, ApJ, 470, 172

Tucker, D. L., et al. 1997, MNRAS, 285, L5 